Short Communication

\title{
Excluded volume effect of counterions and water dipoles near a highly charged surface due to a rotationally averaged Boltzmann factor for water dipoles
}

\author{
Ekaterina Gongadze and Aleš Iglič \\ Laboratory of Biophysics, Faculty of Electrical Engineering, University of Ljubljana, Tržaška cesta 25, Ljubljana, Slovenia
}

\begin{abstract}
Water ordering near a negatively charged electrode is one of the decisive factors determining the interactions of an electrode with the surrounding electrolyte solution or tissue. In this work, the generalized Langevin-Bikerman model (Gongadze-Iglič model) taking into account the cavity field and the excluded volume principle is used to calculate the space dependency of ions and water number densities in the vicinity of a highly charged surface. It is shown that for high enough surface charged densities the usual trend of increasing counterion number density towards the charged surface may be completely reversed, i.e. the drop in the counterions number density near the charged surface is predicted.
\end{abstract}

Key words: Electric double layer - Excluded volume effect - Orientational ordering of water — Finite-sized molecules

The electrically charged surface of the electrode or implant attracts cations and repels anions, and consequently an electric double layer (EDL) is formed (Gouy 1910; Kenkel and Macdonald 1984; McLaughlin 1989; Lamperski and Outhwaite 2002; Butt et al. 2003; Bivas and Ermakov 2007; Bazant et al. 2009; Das et al. 2012). It is generally accepted that negatively charged electrode is covered by a thin molecular layer of water with a net dipole orientation dependent on the respective electrode charge (Bergethon 1998). The adsorbed water molecules are partially displaced by counterions (cations) of EDL formed at the negatively charged electrode surface (Gongadze and Iglič 2012) and specific adsorption and/or chemisorption of coions (anions) (Bergethon 1998; Butt et al. 2003).

The mean-field Langevin Poisson-Boltzmann model for point-like ions and water molecules (Gongadze et al. 2011) was recently modified by including the cavity field (Velikonja et al. 2013). Previously, also the mean-field Langevin-Bikerman model for finite size of molecules (Iglič et al. 2010) was generalized by including the cavity field (Gongadze and Iglič 2012). It was shown that in the vicinity of a charged surface the number densities of counterions $(+)$, coions (-) and water molecules $(\mathrm{w})$ are stronly influenced by the charged surface

Correspondence to: Aleš Iglič, Laboratory of Biophysics, Faculty of Electrical Engineering, University of Ljubljana, Tržaška cesta 25, SI-1000 Ljubljana, Slovenia

E-mail: ales.iglic@fe.uni-lj.si
(Gongadze and Iglič 2012). Within the lattice statistic approach the probabilities that a single lattice site in electrolyte solution is occupied by a particle of one of the three kinds is determined by the corresponding (average) Boltzmann factors (Gongadze and Iglič 2012):

$$
\begin{aligned}
& n_{+}(x)=\frac{n_{0} e^{-e_{0} \phi(x) \beta}}{n_{0} e^{e_{0} \phi(x) \beta}+n_{0} e^{-e_{0} \phi(x) \beta}+n_{0 w}\left\langle e^{-\gamma p_{0} E \beta \cos \omega}\right\rangle_{\omega}} \\
& n_{-}(x)=\frac{n_{0} e^{e_{0} \phi(x) \beta}}{n_{0} e^{e_{0} \phi(x) \beta}+n_{0} e^{-e_{0} \phi(x) \beta}+n_{0 w}\left\langle e^{-\gamma p_{0} E \beta \cos \omega}\right\rangle_{\omega}} \\
& n_{w}(x)=\frac{n_{0 w}\left\langle e^{-\gamma p_{0} E \beta \cos \omega}\right\rangle_{\omega}}{n_{0} e^{e_{0} \phi(x) \beta}+n_{0} e^{-e_{0} \phi(x) \beta}+n_{0 w}\left\langle e^{-\gamma p_{0} E \beta \cos \omega}\right\rangle_{\omega}}
\end{aligned}
$$

where

$$
\begin{aligned}
& \left\langle e^{-\gamma p_{0} E \beta \cos \omega}\right\rangle_{\omega}=\frac{\int e^{-\gamma p_{0} E \beta \cos \omega} \mathrm{d} \Omega}{\int \mathrm{d} \Omega}= \\
& =\frac{2 \pi \int_{\pi}^{0} d(\cos \omega) e^{-\gamma p_{0} E \beta \cos \omega}}{4 \pi}=\frac{\sinh \left(\gamma p_{0} E \beta\right)}{\gamma p_{0} E \beta}
\end{aligned}
$$

and $\mathrm{d} \Omega=2 \pi \sin \omega \mathrm{d} \omega$. Here $n_{+}(x)$ and $n_{-}(x)$ are the number densities of counterions and coions, respectively, $n_{\mathrm{w}}(x)$ is the number density of water molecules, $\phi(x)$ is the electrostatic po- 
tential, $E$ is the magnitude of the electric field strength, $e_{0}$ is the elementary charge, $k T$ is the thermal energy, $\beta=1 / k T, n_{0}$ is the bulk number density of positively and negatively charged ions in electrolyte solution, $n_{0 \mathrm{w}}$ is the bulk number density of water, $n_{\mathrm{S}}$ being the number density of lattice sites: $n_{\mathrm{S}}=n_{\mathrm{W}}(x)+n_{+}(x)$ $+n_{-}(x)$ in bulk $n_{\mathrm{s}}=n_{0 \mathrm{w}}+2 n_{0}, p_{0}$ stands for the magnitude of the water dipole moment and $\gamma=3\left(2+n^{2}\right) / 6$, where $n=1.33$ is the optical refractive index of water (Gongadze and Iglič 2012). The axis $x$ is perpendicular to the charged surface and points in the direction of the bulk solution. Note that $\omega$ describes the angle between the water dipole vector and the vector in the direction of the $x$-axis, i.e. vector -E. The space dependence of electric potential can be determined by solving (Gongadze and Iglič 2012):

$$
\nabla \cdot\left(\varepsilon_{0} \varepsilon_{r}(x) \nabla \phi(x)\right)=-2 e_{0} n_{s} n_{0} \frac{\sinh \left(e_{0} \phi(x) \beta\right)}{\mathrm{D}(\phi(x), E(x))}
$$

where the relative (effective) permittivity is (Gongadze and Iglič 2012):

$$
\begin{aligned}
& \varepsilon_{r}=n^{2}+n_{s} n_{0 w} \frac{p_{0}}{\varepsilon_{0}}\left(\frac{2+n^{2}}{3}\right) \frac{F\left(\gamma p_{0} E(x) \beta\right)}{D(\phi(x), E(x)) E(x)} \\
& \mathrm{F}(u)=(\operatorname{coth}(u)-1 / u) \sinh (u) / u
\end{aligned}
$$
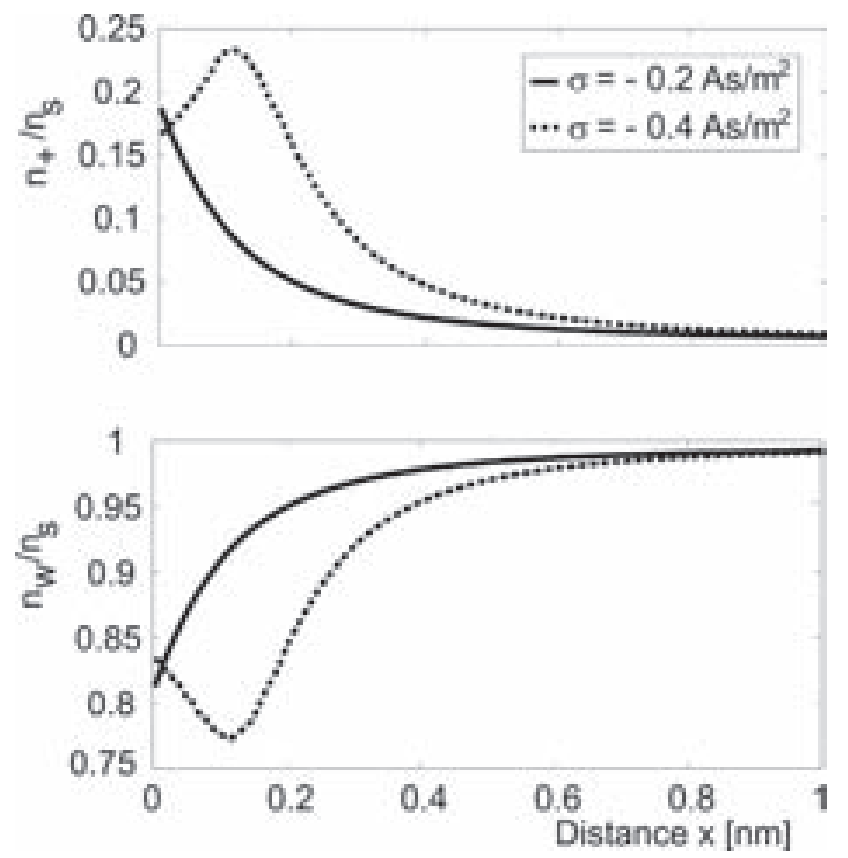

Figure 1. The relative number density of counterions $\left(n_{+}(x) / n_{\mathrm{S}}\right)$ and $\left(n_{\mathrm{w}}(x) / n_{\mathrm{s}}\right)$ as a function of the distance from the charged planar surface for two values of the surface charge density $\sigma=-0.2 \mathrm{As} / \mathrm{m}^{2}$ and $\sigma=-0.4 \mathrm{As} / \mathrm{m}^{2}$. The dipole moment of water $p_{0}=3.1 \mathrm{D}$, the bulk concentration of salt $n_{0} / N_{\mathrm{A}}=0.15 \mathrm{~mol} / \mathrm{l}$ and the bulk water concentration $n_{0 \mathrm{w}} / N_{\mathrm{A}}=55 \mathrm{~mol} / \mathrm{l}$. and

$$
\begin{aligned}
& D(\phi(x), E(x))=2 n_{0} \cosh \left(e_{0} \phi(x) \beta\right)+ \\
& +n_{0 w} \sinh \left(\gamma p_{0} E(x) \beta\right) /\left(\gamma p_{0} E(x) \beta\right)
\end{aligned}
$$

Eq. 5, subject to correponding boundary conditions, was solved numerically for a planar geometry using Finite element method (FEM) within program package Comsol Multiphysics 4.3 Software (COMSOL AB, Stockholm) as described in Gongadze and Iglič (2012). Eqs. 1-8 are not new, but taken from our previous work (Gongadze and Iglič 2012). However, in this short communication we would like to point out to an important role of rotationally averaged Boltzmann factor of water molecules $n_{0 \mathrm{w}}<\exp \left(-\gamma p_{0} E \beta \cos \right.$ $\omega)>_{\omega}$ in ions and water distribution functions (Eqs. 1-3) in the case of a highly charged electrode surface.

Fig. 1 shows the calculated spatial dependences of $n_{+}(x)$ and $n_{\mathrm{w}}(x)$ for two values of the surface charge density $\sigma$. As expected, for $\sigma=-0.2 \mathrm{As} / \mathrm{m}^{2}$ the counterion number density increases in a direction towards the charged surface, whereas the number density of water decreases due to the excluded volume effect (Bikerman 1942; Kralj-Iglič and Iglič 1996; Gongadze and Iglič 2012). However, for $\sigma=-0.4 \mathrm{As} / \mathrm{m}^{2}$ the number density $n_{+}(x)$ attains a maximum close to the charged surface and then starts to decrease. In agreement, $n_{\mathrm{w}}(x)$ attains a minimum at the same location and then increases towards the charged surface. The predicted behaviour of $n_{+}(x)$ and $n_{\mathrm{w}}(x)$ for large magnitudes of $\sigma$ (in Fig. 1 for $-0.4 \mathrm{As} / \mathrm{m}^{2}$ ) can be explained by a strong concomitant increase of $E$ and $n_{0 \mathrm{w}}<\exp \left(-\gamma p_{0} E \beta \cos \omega\right)>_{\omega}$ in the vicinity of the charged surface which prevail over the increasing of the counterion Boltzmann factor $n_{0} \exp \left(-e_{0} \phi(x) \beta\right)$ due to growing $-\phi(x)$ (see Eqs. 1 and 3 ).

In conclusion, Bikerman theory (Bikerman 1942) always predicts depletion of water molecules due to accumulation of counterions (see for example Bazant et al. 2009; Kralj-Iglič and Iglič 1996; Lamperski and Outhwaite 2002). On the contrary, within the generalized Langevin-Bikerman model (Gongadze and Iglič 2012), the partial depletion of water molecules can be reduced at large surface charge densities due to the competition between the counterion Boltzmann factor $n_{0} \exp \left(-e_{0} \phi(x) \beta\right)$ and rotationally averaged water Boltzmann factor $n_{0 \mathrm{w}}<\exp \left(-\gamma p_{0} E \beta \cos \omega\right)>_{\omega}$ in the region near the charged surface.

Acknowledgements. This work was supported by ARRS grants J32120, J1-4109, J1-4136, J3-4108, P2-0232-1538 and Slovene Human Resources Development and Scholarship Fund. We ackowledge the helpful discussion with F. R. Hamou.

\section{References}

Bazant M. Z., Kilic M. S., Storey B., Ajdari A. (2009): Towards an understanding of induced-charge electrokinetics at large ap- 
plied voltages in concentrated solutions. Adv. Colloid Interface Sci. 152, $48-88$ http://dx.doi.org/10.1016/j.cis.2009.10.001

Bikerman J. J. (1942): Structure and capacity of the electrical double layer. Phil. Mag. 33, 384-397

Bivas I., Ermakov Y. A. (2007): Elasticity and electrostatics of amphiphilic layers. In: Advances in Planar Lipid Bilayers and Liposomes. (Ed. A. Leitmannova Liu), Vol. 5, pp. 313-343, Elsevier, Amsterdam

Bergethon P. (1998): The Physical Basis of Biochemistry, Springer, New York, Dordrecht http://dx.doi.org/10.1007/978-1-4757-2963-4

Butt H. J., Graf K., Kappl M. (2003): Physics and Chemistry of Interfaces. Wiley-VCH Verlag, Weinheim http://dx.doi.org/10.1002/3527602313

Das S., Chakraborty S., Mitra S. K. (2012): Redefining electrical double layer thickness in narrow confinements: Effect of solvent polarization. Phys. Rev. E 85, 051508 http://dx.doi.org/10.1103/PhysRevE.85.051508

Gongadze E., van Rienen U., Kralj-Iglič V., Iglič A. (2011): Langevin Poisson-Boltzmann equation: point-like ions and water dipoles near charged membrane surface. Gen. Physiol. Biophys. 30, $130-137$ http://dx.doi.org/10.4149/gpb_2011_02_130

Gongadze E., Iglič A. (2012) : Decrease of permittivity of an electrolyte solution near a charged surface due to saturation and excluded volume effects. Bioelectrochemistry 87, 199-203

http://dx.doi.org/10.1016/j.bioelechem.2011.12.001
Gouy M.G. (1910): Sur la constitution de la charge electrique a la surface d' un electrolyte. J. Physique 9, 457-468

Iglič A., Gongadze E., Bohinc K. (2010): Excluded volume effect and orientational ordering near charged surface in solution of ions and Langevin dipoles. Bioelectrochmistry 79, 223-227 http://dx.doi.org/10.1016/j.bioelechem.2010.05.003

Kenkel S.W., Macdonald J. R. (1984): A lattice model for the electrical double layer using finite-length dipoles. J. Chem. Phys. 81, 3215-3221 http://dx.doi.org/10.1063/1.448028

Kralj-Iglič V., Iglič A. (1996): A simple statistical mechanical approach to the free energy of the electric double layer including the excluded volume. J. Phys. II (France) 6, 477-491 http://dx.doi.org/10.1051/jp2:1996193

Lamperski S., Outhwaite C. W. (2002): Volume term in the inhomogeneous Poisson-Boltzmann theory for high surface charge. Langmuir 18, 3423-3424 http://dx.doi.org/10.1021/la011852v

McLaughlin S. A. (1989): The electrostatic properties of membranes. Rev. Biophys. Chem. 18, 113-136 http://dx.doi.org/10.1146/annurev.bb.18.060189.000553

Velikonja A., Perutková Š., Gongadze E., Kramar P., Polak A., Maček-Lebar A., Iglič A. (2013): Monovalent ions and water dipoles in contact with dipolar zwitterionic lipid headroups theory and MD simulations. Int. J. Mol. Sci. 14, 2846-2861 http://dx.doi.org/10.3390/ijms14022846

Received: November 15, 2012

Final version accepted: December 10, 2012 